\title{
The mass distribution of unstable cores in turbulent magnetized clouds
}

\author{
Paolo Padoan $^{1}$, Åke Nordlund ${ }^{2}$, Alexei G. Kritsuk ${ }^{1}$, \\ Michael L. Norman ${ }^{1}$ and Pak Shing $\mathbf{L i}^{3}$ \\ ${ }^{1}$ Department of Physics, University of California, San Diego, CASS/UCSD 0424, 9500 Gilman \\ Drive, La Jolla, CA 92093-0424, \\ email: ppadoan@ucsd.edu \\ ${ }^{2}$ Astronomical Observatory/NBIfAFG, Juliane Maries Vej 30, DK-2100, Copenhagen, \\ Denmark \\ ${ }^{3}$ Astronomy Department, University of California, Berkeley, CA 94720
}

\begin{abstract}
The predictions of the Padoan and Nordlund IMF model are tested using the largest simulations of supersonic hydrodynamic (HD) and magneto-hydrodynamic (MHD) turbulence to date ( $\sim 1000^{3}$ computational zones). The striking difference between the HD and MHD regimes, predicted by the model, is recovered.
\end{abstract}

Keywords. star formation, IMF, turbulence

\section{Introduction}

The mass distribution of prestellar cores was derived by Padoan \& Nordlund (2002) assuming that: i) the turbulence has a power law energy power spectrum; ii) cores are formed by shocks in the turbulent flow and have size and density scaling as the postshock layer thickness and density; iii) the number of such shocks scales self-similarly as the inverse of the cube of their size; iv) the condition for the collapse of small cores is that they exceed their Bonnor-Ebert mass, derived from the lognormal probability density function (pdf) of the gas density independently of the core mass. After integrating over the probability of exceeding the Bonnor-Ebert mass, the mass distribution is:

$$
N(m) d m=C\left[1+\operatorname{erf}\left(\frac{4 \ln (m)+\sigma^{2}}{2 \sqrt{2} \sigma}\right)\right] m^{-x} d m
$$

where the mass $m=m / m_{\mathrm{BE}, 0}$ is in units of the average Bonnor-Ebert mass,

$$
m_{\mathrm{BE}, 0}=3.3 \mathrm{M}_{\odot}\left(\frac{n_{0}}{10^{3} \mathrm{~cm}^{-3}}\right)^{-1 / 2}\left(\frac{T_{0}}{10 \mathrm{~K}}\right)^{3 / 2},
$$

$\sigma$ is the standard deviation of the gas density pdf (assumed to be a lognormal) related to the rms Mach number of the turbulence (the sonic or the Alfvénic Mach number in the HD or MHD regime respectively, see below):

$$
\sigma=\sqrt{\ln \left(1+M_{0}^{2} / 4\right)}
$$

The coefficient $C$ is not discussed here, but it would be important for modeling the star formation efficiency. The power law slope, $x$, is determined by the power law slope of the energy spectrum, $\beta$ ( $\beta \approx 5 / 3$ in incompressible turbulence and $\beta=2$ in Burgers zero-pressure model), and by the shock jump conditions:

$$
x=3 /(4-\beta)
$$



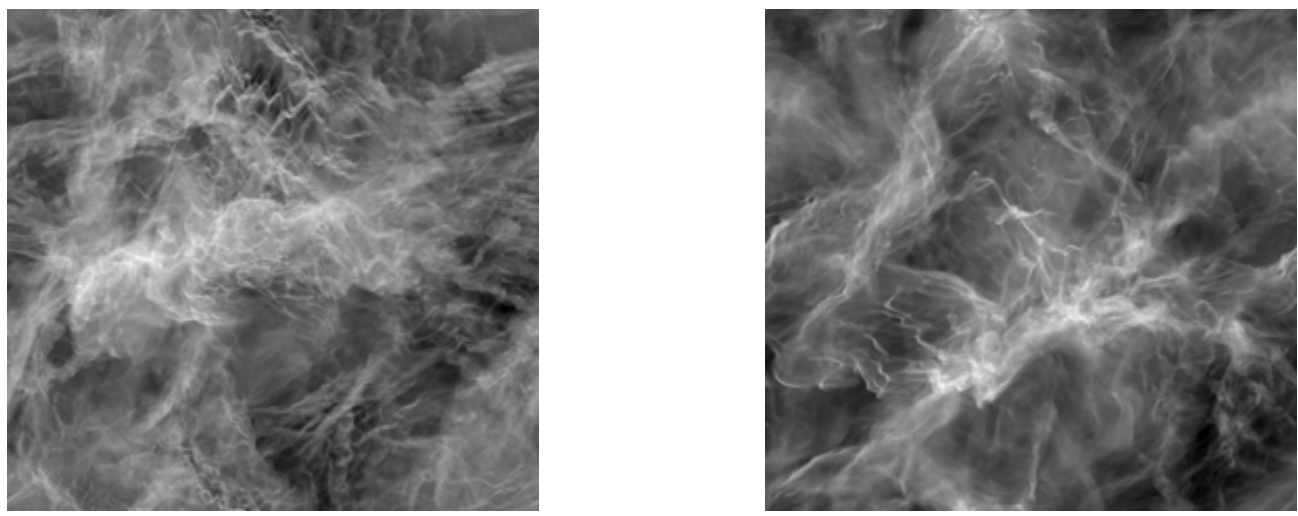

Figure 1. Logarithm of projected density from a snapshot of the Stagger-Code HD run (left) and MHD run (right).

for $B \geqslant B_{\text {cr }}$ (MHD jump conditions), and

$$
x=3 /(5-2 \beta)
$$

for $B<B_{\text {cr }}$ (isothermal HD jump conditions). The critical magnetic field value that separates the two regimes is given by the condition that the postshock gas pressure is of order the postshock magnetic pressure, corresponding to an rms Alfvénic Mach number, $M_{\mathrm{A}}$, of the order of the ratio of the mean gas and magnetic pressures, $M_{\mathrm{A}} \sim P_{\mathrm{g}} / P_{\mathrm{m}}$. This condition gives

$$
B_{\mathrm{cr}} \approx 3 \mu \mathrm{G}\left(\frac{T_{0}}{10 \mathrm{~K}}\right)\left(\frac{u_{0}}{1 \mathrm{~km} / \mathrm{s}}\right)^{-1}\left(\frac{n_{0}}{10^{4} \mathrm{~cm}^{-3}}\right)
$$

Because the Galactic magnetic field strength is locally $6 \pm 2 \mu \mathrm{G}$ (Beck 2001; Han, Ferrière, \& Manchester 2004), and most likely larger in prestellar cores (Crutcher 1999; Bourke et al. 2001), current star formation in the galactic disk is in the MHD regime. For a value of $\beta=1.9$, typical of supersonic turbulence, $x=1.4$, similar to the Salpeter slope of the stellar IMF $(x=1.35$, Salpeter 1955). For very weak magnetic fields, perhaps in protogalaxies at very large redshifts, the slope is $x=2.5$, assuming again $\beta=1.9$. At high redshifts the temperature may also be larger, $T>100 \mathrm{~K}$ (e.g. Palla, Salpeter, \& Stahler 1983; Abel, Bryan \& Norman 2000), giving an even larger value of $B_{\text {cr }}$.

The peak of the distribution shifts to smaller masses with increasing Mach number and gas density, increasing the abundance of low mass stars and brown dwarfs as well. This mass distribution matches very well the observed stellar IMF of Chabrier (2003), for reasonable physical parameters, suggesting that the process of turbulent fragmentation may play a major role in the origin of the stellar IMF (Padoan \& Nordlund 2002), with only minor effects due to gravitational fragmentation, accretion or merging.

We must stress the statistical nature of the origin of the mass distribution. The present model is based on the statistics of turbulence, and the scaling laws are averages over a large sample. But massive stars originate from shocks on the largest scales, and are rare because such large scale shocks are rare. Being rare, these shocks may deviate from the average properties of the turbulence, resulting in fluctuations of the number of massive stars in different star-forming environments, in excess of the Poisson variance. Large variations of observed mass distributions from place to place are evidence of the stochastic nature of the process of star formation, although this process is universal. 

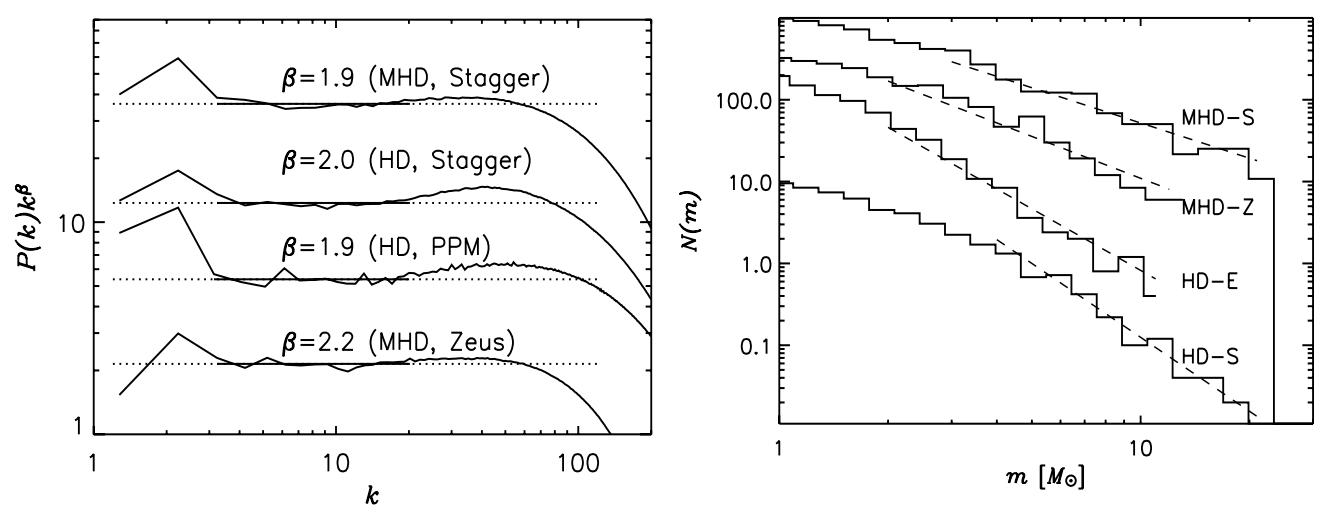

Figure 2. Left: Compensated power spectra of the main four runs and least square fits in the range of wavenumbers $3 \leqslant k \leqslant 20$. Right: Mass distributions of gravitationally unstable cores above $1 \mathrm{M}_{\odot}$, for the main four experiments scaled to a mean density of $10^{4} \mathrm{~cm}^{-3}$, a box size of $6 \mathrm{pc}$, and a clumpfind density resolution $f=8 \%$. The dashed lines show the power law derived from the power spectrum slope and the shock jump conditions of the corresponding simulations, according to the turbulent fragmentation model. The histograms are arbitrarily offset in the vertical direction for clarity.

\section{The simulations}

Our turbulent fragmentation model outlined in the previous section neglects gravity, so we test it with numerical simulations of supersonic MHD turbulence without self-gravity. The main comparison between the MHD and HD regimes is carried out with the Stagger Code, on a numerical mesh of $1,000^{3}$ computational zones. We also simulated the HD regime with the Enzo code (Norman \& Bryan 1999), and the MHD regime with the Zeus code (Stone \& Norman 1992), in both cases on a numerical mesh of $1,024^{3}$ computational zones.

In all simulations we used periodic boundary conditions, isothermal equation of state, random forcing in Fourier space at wavenumbers $k \leqslant 2(k=1$ correspond to the computational box size), uniform initial density and magnetic field (in the MHD runs), random initial velocity field with power only at wavenumbers $k \leqslant 2$. The results of this work are for rms Mach number 10, unless otherwise specified. In Figure 1 we show two projections of the density field, from the HD and MHD Stagger Code runs. The density field in the HD run appears to be significantly more fragmented than its MHD counterpart. This is due to the fact that i) The density contrast in the HD shocks is larger than in the MHD shocks, creating thinner postshock layers from shocks with equal sonic Mach number; ii) the HD postshock layers are Kelvin-Helmholtz unstable, due to the strong shear flow that originates in oblique shocks, while in most of the MHD layers the same instability is suppressed by the magnetic field that is amplified in the compression.

Figure 2 (left panel) shows the compensated power spectra of the four main simulations. The power spectra are defined as the squared of the modulus of the Fourier transform of the velocity, integrated over a wave-number shell. If $\hat{\mathbf{u}}_{i}(\mathbf{k})$ is the Fourier transform of the $i$ velocity component, $\mathbf{u}_{i}(\mathbf{r})$, the power spectrum is $P_{i}(k)=\sum \hat{\mathbf{u}}_{i} \hat{\mathbf{u}}_{i}^{*}$, where the sum is over all $i$ and all wave-numbers $\mathbf{k}$ in the shell $k \leqslant|\mathbf{k}|<k+d k . P(k)$ is proportional to the contribution to the mean square velocity from all wave-numbers in the shell $k \leqslant$ $|\mathbf{k}|<k+d k$.

The plots in Figure 2 have been arbitrarily shifted in the vertical direction. Deviations of more than a factor of two from a power law fit are found only at wavenumbers $k>100$, so the turbulence is roughly scale-free for almost two orders of magnitude 

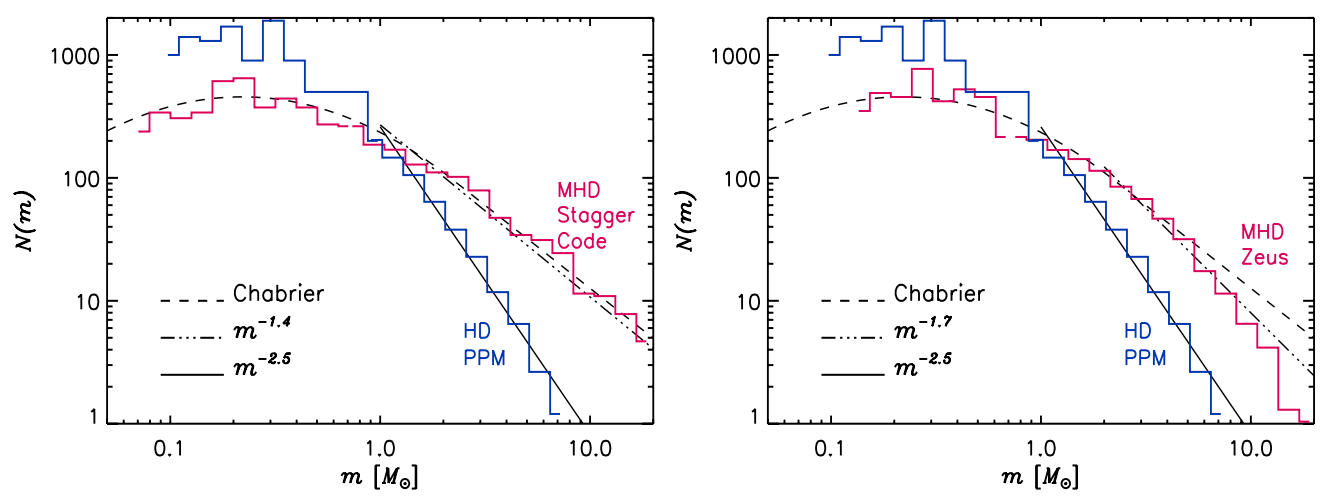

Figure 3. Left: Mass distributions of gravitationally unstable cores from the HD and MHD regimes (Enzo and Stagger Code respectively), computed with $f=16 \%$ and assuming a mean gas density of $10^{4} \mathrm{~cm}^{-3}$. Each mass distribution is the result of matching two mass distributions, computed for a computational box size of 1 pc and 6 pc. The Chabrier IMF (Chabrier 2003) and the fragmentation model predictions for the power law mass distributions of each run are also plotted. Right: Same as left, but for the Zeus MHD run. Notice how the steeper Zeus mass distribution is recovered. The model predicts the Zeus mass distribution to be steeper than the Stagger-Code one, as a result of the steeper turbulence power spectrum in the more diffusive Zeus run.

in wavenumbers. We have chosen to measure the power spectrum slope in the range $3 \leqslant k \leqslant 20$, because larger wavenumbers are affected by the bottleneck effect (e.g. Falkovich 1994; Dobler et al. 2003; Haugen \& Brandenburg 2004). We get $\beta=1.9$ and 2.0 from the Stagger code in the MHD and HD regimes respectively. The Enzo code in the $\mathrm{HD}$ regime gives $\beta=1.9$, and Zeus in the MHD regime $\beta=2.2$. The corresponding values of the exponent of the power law range of the mass distribution of unstable cores are, according to the model of turbulent fragmentation, $x=1.4$ and 3 for the MHD and HD regimes of the Stagger code, and $x=1.7$ and 2.5 for the MHD and HD regimes of Zeus and Enzo respectively.

If the power spectrum is not converged to its correct slope, due to a lack of dynamic range of scales or an excess of numerical diffusivity, the slope of the mass distribution may be strongly affected. For example, in the MHD case we get $x=1.4$ from the Stagger code, and $x=1.7$ from the Zeus code. Padoan et al. (2006) have recently obtained a measurement of the velocity power spectrum in the Perseus molecular cloud complex. Their result is $\beta=1.81 \pm 0.10$, which rules out the larger power spectrum slopes generated by more dissipative SPH simulations ( $\operatorname{see} \S 4$ ).

\section{Mass distributions}

We compute the mass distribution of gravitationally unstable cores formed in turbulence simulations without self-gravity in order to learn about the effect of turbulence, and to compare with the predictions of the turbulent fragmentation model. Our results are obtained after the driven turbulence has statistically relaxed, which could not be achieved with self-gravity. The mass distributions derived in this work and the mass distribution predicted by Padoan \& Nordlund (2002), should be considered as a guess of the final outcome of more realistic simulations with self-gravity. In simulations including self-gravity the mass distribution of unstable cores may initially vary with time, as the most massive cores are still being assembled by converging turbulent flows while their 

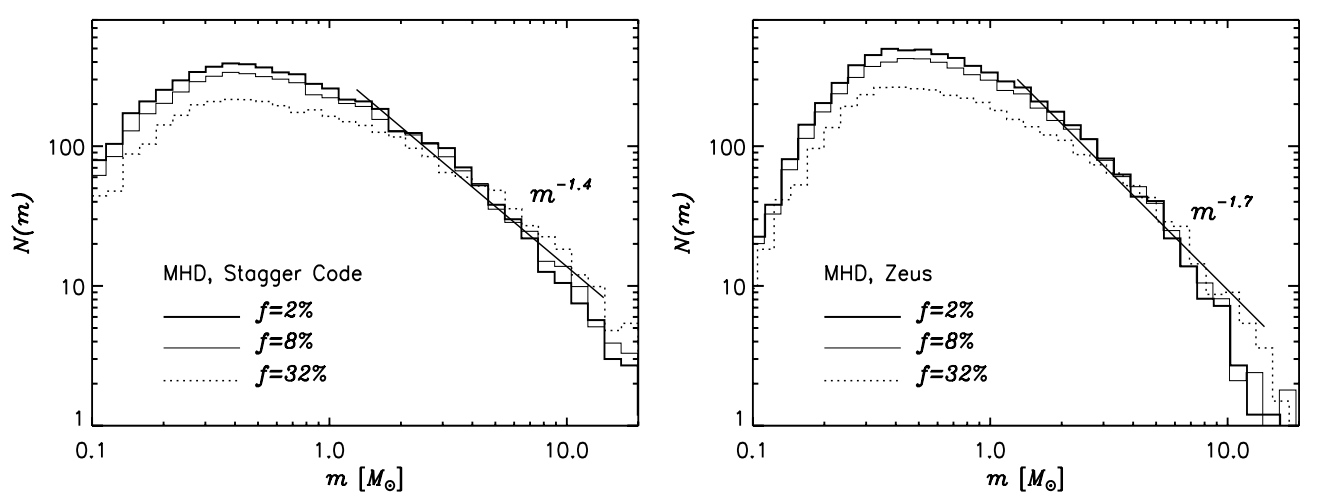

Figure 4. Left: Test of convergence of the mass distribution with decreasing value of the density resolution parameter, $f$, from the Stagger-Code MHD run. The mass distribution is well converged at $f=8 \%$. Right: Same as left, but from the Zeus MHD run.

central part has already collapsed, while in the present study the mass distribution has no time dependence.

Cores are defined as connected overdensities that cannot be split into two or more overdensities of amplitude $\delta \rho / \rho>f$. The unstable cores are the cores with mass larger than their Bonnor-Ebert mass. Our clumpfind algorithm scans the density field with discrete density levels, each of amplitude $f$ relative to the previous one. Only the connected regions above each density level that are larger than their Bonnor-Ebert mass are selected as unstable cores. After this selection, the unstable cores from all levels form a hierarchy tree. Only the final (unsplit) core of each branch is retained.

Different clumpfind algorithms treat the mass surrounding the cores in different ways. The algorithm by Williams, de Geus, \& Blitz (1994) uses up all the available mass (see their Figure 2). This results in a core formation efficiency of $100 \%$ above the threshold density. Our algorithm, instead, assigns to each core only the mass within the density isosurface that defines the core (below that density level the core would be merged with its next neighbor). With our choice, the smallest possible mass is assigned to each core. With this algorithm, and with conditions typical of molecular clouds, the unstable cores contain a few percent of the total mass, in agreement with the star formation efficiency in molecular clouds.

In the right panel of Figure 2, the mass distributions above $1 \mathrm{M}_{\odot}$ are plotted for the main four experiments scaled to a mean density of $10^{4} \mathrm{~cm}^{-3}$, a box size of $6 \mathrm{pc}$, and a clumpfind density resolution $f=8 \%$. Overplotted on the corresponding power law section of each mass distribution, the dashed lines show the power law derived from the power spectrum slope and the shock jump conditions of each simulation, according to the turbulent fragmentation model, $x=3 /(4-\beta)$ in the MHD regime, and $x=3 /(5-2 \beta)$ in the HD regime. The general trend is recovered well, despite deviations to be expected because this mass distributions are from single snapshots, not time averages.

Figure 3 shows the mass distributions of the HD and MHD regimes, computed with $f=16 \%$ and assuming a mean gas density of $10^{4} \mathrm{~cm}^{-3}$. Each mass distribution is the result of matching two mass distributions, computed for a box size of $1 \mathrm{pc}$ and $6 \mathrm{pc}$. In the $6 \mathrm{pc}$ case we can sample masses in the range $1-10 \mathrm{M}_{\odot}$, and probe the effect of the turbulence power spectrum and shock jump conditions on the mass distribution, but the mass distribution is incomplete for stars below $1 \mathrm{M}_{\odot}$. The 1 pc case samples well the turnover region, defining the peak mass for that mean density and rms Mach number, 

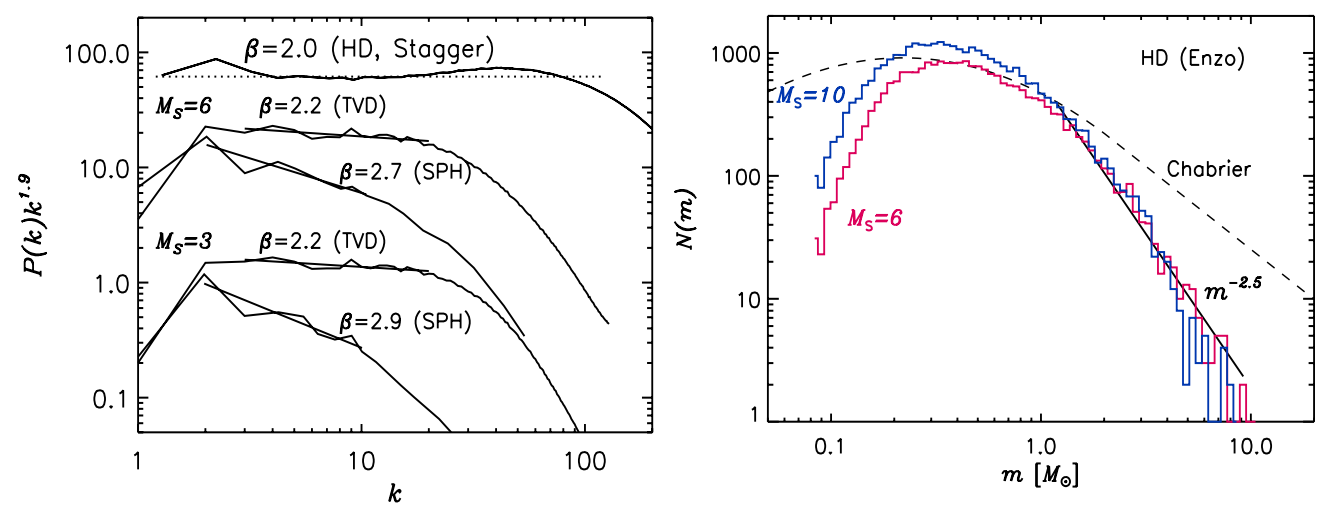

Figure 5. Left: Power spectra compensated for the slope of the Stagger-Code HD run, $\beta=1.9$. The TVD and SPH power spectra are the same as in Figure 2 of Ballesteros-Paredes et al. (2006), for the Mach numbers 3 and 6. Right: Mass distributions of gravitationally unstable cores from the Enzo HD runs with $M_{\mathrm{S}}=6$ and $M_{\mathrm{S}}=10, f=8 \%$ and assuming a mean gas density of $10^{4} \mathrm{~cm}^{-3}$ and a box size of 6 pc. Each mass distribution contains unstable cores from two snapshots. The Chabrier (2003) IMF (dashed line) and the power law predicted by the turbulent fragmentation model (solid line) are also plotted.

but does not yield intermediate and high mass stars. The numerical mass distributions reproduce the difference between the HD and MHD regimes predicted by the analytical model. The steeper mass distribution expected from the Zeus run, compared with the Stagger Code run, due to the steeper Zeus turbulence power spectrum, is also recovered. Furthermore, the mass distribution of the MHD regime is consistent with Chabrier's stellar IMF (Chabrier 2003).

Once the physical size and mean density of the system are chosen, the clumpfind algorithm depends only on two parameters: i) The spacing of the discrete density levels, $f$, and ii) the minimum density above which cores are selected, $\rho_{\min }$. Our results do not change significantly for values of $\rho_{\text {min }}$ below the mean gas density, so we scan the density field only above the mean density. We have also verified the convergence of the mass distribution with decreasing values of $f$. The convergence is typically obtained already at a value of $f \approx 16 \%$. In Figure 4 we plot the mass distributions of the MHD regime assuming a mean gas density of $10^{4} \mathrm{~cm}^{-3}$ and a 6 pc size. Between $f=32 \%$ and $f=8 \%$ there is a tendency to fragment the largest cores and create a larger number of small cores. However, the differences between $f=8 \%$ and $f=2 \%$ are small and the slope of the mass distribution above $2-3 \mathrm{M}_{\odot}$ is independent of resolution.

\section{Discussion}

Using TVD and SPH simulations, Ballesteros-Paredes et al. (2006) conclude that i) the core mass distribution depends on the rms Mach number and ii) it is not a power law, even at large masses. The first statement is in part correct. The Padoan and Nordlund model contains a Mach number dependence, with the peak of the mass distribution shifting to lower masses as the Mach number increases, in agreement with the numerical results in Ballesteros-Paredes et al. (2006). However, in the model the mass distribution above the peak is a power law with slope independent of the Mach number. Based only on the TVD simulations of Ballesteros-Paredes et al. (2002), and accounting for their relative low numerical resolution, there is actually no contradiction with our results (see 
their Figure 4). Significant differences arises only from their SPH simulations (see their Figure 5), but these simulations fail to produce a realistic velocity power spectrum.

The left panel of Figure 5 compares the power spectrum from the Stagger-Code HD run with two TVD and two SPH power spectra from Ballesteros-Paredes et al. (2006), for Mach numbers 3 and 6 . The inertial range in both the TVD and SPH cases is not very extended, due to the low numerical resolution. The TVD code gives a slope of $\beta \approx 2.2$, as in our Zeus run, for both Mach numbers. The extent of the inertial range in the TVD run is also comparable to that in Zeus at the same resolution. The power spectra of the SPH runs are instead much steeper, and their slope increases with decreasing Mach number, $\beta \approx 2.7$ for $M_{\mathrm{S}} \approx 6$ and $\beta \approx 2.9$ for $M_{\mathrm{S}} \approx 3$. As confirmed by the TVD runs, the power spectrum should not vary much with Mach number between $M_{\mathrm{S}}=6$ and $M_{\mathrm{S}}=3$. For even lower Mach numbers, the power spectrum should become shallower, and converge to the Kolmogorov value of $\beta \approx 5 / 3$ for $M_{\mathrm{S}}<1$. The dependence of the slope of the SPH power spectrum on the Mach number is therefore both too strong and in the wrong direction. The absence of an inertial range with a reasonable slope or with the correct Mach number dependence, makes the SPH simulations inadequate for testing the turbulent fragmentation model, because the model relies on the scale-free nature of turbulent flows.

The issue of the Mach number dependence, is tested in the right panel of Figure 5, showing the mass distributions from the Enzo HD runs with $M_{\mathrm{S}}=6$ and $M_{\mathrm{S}}=10$ and with $f=8 \%$, assuming a mean gas density of $10^{4} \mathrm{~cm}^{-3}$ and a box size of $6 \mathrm{pc}$. The Figure shows that the power law part of the mass distribution, above $1-2 \mathrm{M}_{\odot}$, is independent of the Mach number and matches the prediction of the turbulent fragmentation model, that is $k^{-2.5}$ for the power spectrum slope $\beta=1.9$ of the HD Enzo runs.

\section{Conclusions}

We have tested the turbulent fragmentation model of Padoan and Nordlund (2002) with the largest numerical simulations of supersonic MHD and HD turbulence to date. The simulations have confirmed the theoretical prediction that the HD regime should yield a much steeper mass distribution of unstable cores than the MHD regime. This result shows that even rather weak magnetic fields (super-Alfvénic turbulence) can be crucial in setting the initial conditions for star formation. Furthermore, star formation at very high redshift may occur in the HD regime, due to the weak magnetic field and to the larger value of the critical magnetic field strength at larger temperatures. If so, the stellar IMF at high redshift may have a much steeper slope above the peak than in present-day star formation.

Numerical simulations can quantitatively account for the role of the turbulence in setting the initial conditions for star formation only if they generate an inertial range of turbulence. This requires both low numerical diffusivity and large numerical resolution. To model present-day star formation that occurs in the MHD regime, the magnetic field cannot be neglected, even if the turbulence is assumed to be super-Alfvénic. SPH simulations of star formation have too large numerical diffusivity, too low numerical resolution and no magnetic fields. This should cast doubts on the value of comparing their predictions with observational data (see also Agertz et al. 2006).

The mass distribution of unstable cores found in the MHD simulations is indistinguishable from the Chabrier stellar IMF (Chabrier 2003) and in agreement with the observed mass distributions of prestellar cores. Such a coincidence may indicate that gravitational fragmentation, competitive accretion or merging, all absent in these turbulence simulations, may not play a major role in the origin of the stellar IMF. 


\section{Acknowledgements}

This research was partially supported by a NASA ATP grant NNG056601G, by an NSF grant AST-0507768, and by a NRAC allocation MCA098020S. We utilized computing resources provided by the San Diego Supercomputer Center, by the National Center for Supercomputing Applications and by the NASA High End Computing Program.

\section{References}

Abel, T., Bryan, G. L. \& Norman, M. L. 2000, ApJ 540, 39

Agertz, O., et al. 2006, astro-ph/0610051

Ballesteros-Paredes, J., Gazol, A., Kim, J., Klessen, R. S., Jappsen, A.-K. \& Tejero, E. 2006, ApJ 637, 384

Beck, R. 2001, Space Science Reviews 99, 243

Chabrier, G. 2003, PASP 115, 763

Dobler, W., Haugen, N. E., Yousef, T. A. \& Brandenburg, A. 2003, Phys. Rev. Let. 68, 026304

Falkovich, G. 1994, Physics of Fluids 6, 1411

Han, J. L., Ferrière, K. \& Manchester, R. N. 2004, ApJ 610, 820

Haugen, N. E. \& Brandenburg, A. 2004, Phys. Rev. E 70, 026405

Norman, M. L. \& Bryan, G. L. 1999, ASSL Vol. 240: Numerical Astrophysics 19

Padoan, P. \& Nordlund, Å. 2002, ApJ 576, 870

Padoan, P., Juvela, M., Kritsuk, A. \& Norman, M. L. 2006, ApJ in press

Palla, F., Salpeter, E. E. \& Stahler, S. W. 1983, ApJ 271, 632

Salpeter, E. E. 1955, ApJ 121, 161

Stone, J. M. \& Norman, M. L. 1992, ApJS 80, 791

Williams, J. P., de Geus, E. J. \& Blitz, L. 1994, ApJ 428, 693

\section{Discussion}

BLITZ: The agreement of the slope and mass range of your simulations with observations is impressive. How good is the normalization? That is, how much gas do you need to form a certain integrated mass of stars?

PADOAN: Yes! The typical star formation efficiency we get with the clump find algorithm is $\sim 5 \%$, similar to the value in molecular clouds. This results from the fact that turbulence puts a fraction of the mass at high density in a small volume (the log normal pdf of gas density), and that mass fraction is a few per cent. The rest of the mass is kept at lower density and higher velocity (until it hits a shock), and cannot take part to the process of star formation.

OstRIKER: You emphasized that observed structure as well as structure in simulations is hierarchical, in the sense that cores form within clumps within filaments within larger filaments. But your model for the power spectrum does not include any aspects of hierarchy in density structure. Could you comment?

PADOAN: The hierarchical structure is included (albeit in a simple fashion) in the hypothesis of self-similarity. Also, the model does not exclude that a small filament is inside a larger one; it does not take advantage of that, because it more simply assumes self-similarity.

Whitworth: (i) How does nature choose the correct combination of $\mathrm{M}_{\mathrm{A}}$ and average density to give an approximately universal ratio of brown dwarfs to H-burning stars; (ii) If all massive cores collapse (both compact and extended ones), one would expect high 
mass stars to be more widely distributed, but this is not what we see. We need some predictions of the distribution of stars as a function of mass.

PADOAN: (i) If you assume Larson's scaling relations you find that the turbulent fragmentation model produces a mass distribution weakly varying with environment. From this point of view, your question may be: what produces the Larson relation? (ii) I don't think this is necessarily the implication of the model but we are starting simulations with sink particles to address this question.

BONNELL: If turbulence gives the upper-mass IMF, then your massive clumps should show the clustering properties of massive stars. This can be used as a strong test of turbulent driven IMFs.

PADOAN: In principle this is a good test. However, observed stellar populations are always dynamically evolved relative to the original star-forming cores, so we will do this comparison as soon as we will follow the collapse of the cores and capture the stars numerically with sink particles. In other words, we will compare stars with stars, not cores with stars.

ANDRE: In a recent paper, Tilly \& Pudritz (2004) published purely hydrodynamical simulations of cluster formation and claimed to find a core mass spectrum with a Salpeter slope at the high-mass end. This is in apparent contradiction with your point that turbulent fragmentation leads to a Salpeter core mass spectrum only in the MHD case and to a steeper spectrum in the HD case. Would you care to comment on what might explain this apparent contradiction?

PADOAN: These were much lower resolution simulations than ours. At that resolution, you cannot resolve well the Salpeter range. In fact, you can see from their IMF plots that the statistical significance of the IMF power law fit in the Salpeter range is very low. 